\title{
Subfrontal trans-lamina terminalis approach to a third ventricular craniopharyngioma
}

\author{
Omar Choudhri, MD, ${ }^{1}$ and Steven D. Chang, MD² \\ 1Department of Neurosurgery, University of California, San Francisco; and \\ ${ }^{2}$ Stanford University Medical Center, Department of Neurosurgery, Stanford, California \\ Craniopharyngiomas are benign, partly cystic epithelial tumors that can rarely occur in a retrochiasmatic location \\ with involvement of the third ventricle. The lamina terminalis is an important neurosurgical corridor to these cranio- \\ pharyngiomas in the anterior portion of the third ventricle. We present a video case of a large midline suprasellar and \\ third ventricular craniopharyngioma in a 32-year-old male with visual disturbances. The tumor was approached with a \\ subfrontal translamina terminalis exposure, and a gross-total resection of the tumor was achieved. This surgery involved \\ working through a lamina terminalis fenestration around the optic nerve, optic chiasm, optic tracts, and the anterior com- \\ municating artery complex. This video illustrates the techniques employed in performing a transbasal anterior skull base \\ approach to the third ventricle and demonstrates vivid surgical anatomy of neurovascular structures around the lamina \\ terminalis.
}

The video can be found here: https://youtu.be/fCYMgx8SnKs.

KEYWORDS lamina terminalis; third ventricle; subfrontal; craniophayngioma; microsurgery; video 